\title{
Estudo coorte de avaliação vocal em sujeitos com Esclerose Múltipla
}

\author{
Cohort study of vocal evaluation in subjects \\ with Multiple Sclerosis
}

\section{Estudio de cohorte de evaluación vocal en sujetos con esclerosis múltiple}

\author{
Raí dos Santos Santiago ${ }^{1}$, Carolina Fiorin Anhoque ${ }^{2}$
}

\begin{abstract}
1.Fonoaudiólogo e Pós-Graduando em Neurociências aplicadas à Reabilitação na Universidade Federal do Rio de Janeiro - UFRJ. Rio de Janeiro-RJ, Brasil.

2.Professora Adjunta do Departamento de Fonoaudiologia da Universidade Federal do Espírito Santo UFES. Vitória-ES, Brasil.
\end{abstract}

\begin{abstract}
Resumo
Objetivo. Caracterizar o impacto vocal de sujeitos com esclerose múltipla no curso da evolução da doença em sua qualidade de vida relacionada à voz, identificando os sintomas vocais, além de sintomas de ansiedade, depressão e fadiga vocal. Método. Foram recrutados 15 sujeitos de ambos os gêneros que frequentam o ambulatório de neurologia do Hospital Universitário Cassiano Antonio Moraes. Os sujeitos foram avaliados independentes da presença de surto desmielinizante para realizar avaliação perceptivo-auditiva da voz, Qualidade de Vida em Voz, Índice de Desvantagem Vocal, Índice de Fadiga Vocal, Inventário de Ansiedade e Depressão de Beck em diferentes momentos, repetindo-se o protocolo proposto. Resultados. A comparação dos dados de auto-avaliação após cinco meses mostraram modificações nos protocolos IDV $(p=0,008), Q V V(p=0,00, p=0,012, p=0,00)$, IFV $(p=0,000, p=0,003)$ e avaliação perceptivo-auditiva nos domínios Astenia, Tensão e Instabilidade $(p=0,002, p=0,000$ e $p=0,000)$. Houve permanência do quadro de limitação e fala lenta na reavaliação e, os sujeitos passaram a apresentar sintomas sensitivos, o que não era observado no primeiro contato. Conclusão. Foi detectado que há alteração vocal principalmente manifestada por astenia, tensão e instabilidade. Os sujeitos apresentam impacto na qualidade de vida em voz, na fadiga vocal nos domínios fadiga e restrição vocal e desconforto físico associado à voz, e piora da desvantagem vocal ao longo do curso da doença.

Unitermos. Esclerose múltipla; Disfonia; Qualidade de vida; Fadiga; Transtorno do humor
\end{abstract}

\begin{abstract}
Objective. To characterize the vocal impact of subjects with multiple sclerosis in their voicerelated quality of life, identifying vocal symptoms, as well as symptoms of anxiety, depression and vocal fatigue. Method. Were recruited fifteen subjects from both genders who attended the HUCAM neurology outpatient clinic. The subjects were evaluated independent of the presence of a demyelinating outbreak to perform perceptual-auditory assessment of voice, Quality of Life in Voice, Vocal Disadvantage Index, Vocal Fatigue Index, Beck Anxiety Inventory and Beck Depression Inventory at different times, repeating the proposed protocol. Results. The comparison of the self-assessment data after Five months showed statistically significant changes in the IDV, QVV, IFV and perceptual-auditory evaluation protocols in the Asthenia, Strain and Instability domains. There was continuation of the limitation and slow talk in there evaluation, and the subjects began to present sensory symptoms, which was not observed in the first contact. Conclusion. it was detected that there is vocal alteration mainly manifested by asthenia, strain and instability. The subjects present an impact on voice quality of life, vocal fatigue in the domains of fatigue and vocal restriction and physical discomfort associated with voice, and worsening of vocal disadvantage throughout the course of the disease.
\end{abstract}

Keywords. Multiplesclerosis; Dysphonia; Quality of life; Fatigue; Mooddisorders 


\begin{abstract}
Resumen
Objetivo. Caracterizar el impacto vocal de los sujetos con esclerosis múltiple en el curso de la evolución de la enfermedad en su calidad de vida relacionada con la voz, identificando sintomas vocales, así como síntomas de ansiedad, depresión y fatiga vocal. Método. Se reclutaron quince sujetos de ambos sexos que acudían a la clínica ambulatoria de neurología del Hospital Universitario Cassiano Antonio Moraes. Los sujetos fueron evaluados independientemente de la presencia de un brote desmielinizante para realizar una evaluación de voz auditiva-perceptiva, calidad de vida de voz, índice de discapacidad de voz, índice de fatiga de voz, inventario de ansiedad y depresión de Beck en diferentes momentos, repitiendo el protocolo propuesto. Resultados. La comparación de los datos de autoevaluación después de cinco meses mostró cambios estadísticamente significativos en IDV $(p=0008)$, QVV $(p=0,00, p=0,012, p=0,00)$, protocolos IFV $(p=0,000, p=0,003)$ y evaluación. percepción auditiva en los dominios de astenia, tensión e inestabilidad $(p=0,002$, $p=0,000$ y $p=0,000)$. La limitación y el habla lenta permanecieron en la reevaluación y los sujetos comenzaron a presentar síntomas sensoriales, que no se observaron en el primer contacto. Conclusión. se detectó una alteración vocal que se manifiesta principalmente por astenia, tensión e inestabilidad. Los sujetos tienen un impacto en la calidad de vida de la voz, fatiga vocal en los dominios fatiga y restricción vocal y molestias físicas asociadas con la voz, y empeoramiento de la desventaja vocal a lo largo de la enfermedad.
\end{abstract}

Palabras clave. Esclerosis múltiple; Disfonía; Calidad de vida; Fatiga; Trastorno del estado de ánimo

Trabalho realizado na Universidade Federal do Espírito Santo - UFES. Vitória-ES, Brasil.

\title{
INTRODUÇÃO
}
A Esclerose Múltipla
(EM) é uma doença crônica imunomediada, inflamatória e degenerativa, caracterizada por lesões desmielinizantes do sistema nervoso central (SNC) disseminadas no tempo e no espaço e que, em geral, se manifesta clinicamente por períodos de agudização e remissão, causando graus diversos de incapacidade neurológica ${ }^{1}$. As lesões afetam predominantemente a região periventricular, nervo óptico e a medula espinhal ${ }^{2}$. A prevalência da EM difere em várias partes do mundo, havendo áreas de baixa, média e alta prevalência. As áreas geográficas de alto risco são aquelas com mais de 
30 casos por 100.000 habitantes, de risco médio de 5 a 29 casos por 100.000 habitantes e, de baixo risco, com menos de cinco casos por 100.000 habitantes $^{3}$. No Brasil, tem sido encontrado prevalência média da doença com taxa de 15 a 20 casos por 100.000 habitantes $^{4-6}$.

A doença em geral se inicia entre 20 e 30 anos de idade e apresenta maior prevalência em mulheres ${ }^{7}$, entretanto a causa da EM ainda não é conhecida. Acreditase numa possível interação de fatores genéticos, infecciosos e ambientais no desencadeamento de uma resposta imunológica anormal e, consequentemente, lesão mielínica e dano axonal. Os sintomas da EM estão relacionados às lesões de substância branca do cérebro, nervos ópticos, cerebelo, tronco encefálico ou medula espinhal e, por isso, o quadro clínico é muito variável. Estas lesões podem permanecer estáveis ou progredir com ou sem surtos clínicos ${ }^{7}$.

A EM pode apresentar-se na forma recorrenteremitente (EMRR), secundariamente progressiva (EMSP), primariamente progressiva (EMPP) e progressiva recorrente (EMPR). A progressão da EM pode ser medida pela Escala Expandida de Incapacidades (Expanded Disability Status Scale - EDSS) que utiliza critérios estabelecidos para avaliar sequelas físicas. Esta escala é amplamente utilizada na clínica médica e assistencial, pois fornece pontuação objetiva relativa à severidade dos sintomas apresentados em cada sistema investigado. 
O presente estudo tem como objetivo caracterizar o impacto vocal de sujeitos com esclerose múltipla, durante o curso da doença, em sua qualidade de vida relacionada à voz, identificando os sintomas vocais, além de sintomas de ansiedade, depressão e fadiga vocal.

\section{MÉTODO}

\section{Amostra}

Trata-se de um estudo coorte, aprovado pelo Comitê de Ética em Pesquisa da Instituição de Origem, com o parecer número 2.083.799.

Os sujeitos do estudo foram selecionados de forma aleatória em acordo com os critérios de inclusão. Todos aprovaram a realização da pesquisa e a divulgação dos resultados por meio da assinatura do Termo de Consentimento Livre e Esclarecido, que detalhou os objetivos do estudo e garantiu sigilo dos dados pessoais. Participaram do estudo 15 sujeitos, com idade entre 18 e 63 anos (mediana 37,0 13,2 ), diagnosticados seguindo os Critérios de McDonald e vinculados ao Serviço de Neurologia do Hospital Universitário Cassiano Antonio Moraes (HUCAM-UFES).

Foram considerados como critérios de inclusão os indivíduos com idade superior a 18 anos, EDSS de 0 a 7 e diagnóstico de EM e, como critério de exclusão, ser profissional da voz, apresentar outras alterações 
neurológicas, ser classificado com EDSS entre 8 a 10 e ser tabagista.

\section{Procedimentos}

As coletas de dados sociais e da qualidade vocal foram realizadas no primeiro contato (T1) e após um período de cinco meses (T2), independentes da presença de surto desmielinizante. Todos os protocolos são validados e amplamente utilizados em pesquisa e na clínica fonoaudiológica.

Para a análise da qualidade vocal, cada sujeito era solicitado a emitir a vogal /a/ de forma sustentada, contar os números de 1 a 10 e discorrer sobre algum aspecto do seu dia-a-dia. As análises foram realizadas no momento das emissões e gravadas conforme critérios de avaliação vocal. Foi utilizado à escala de avaliação numérica GRBASI em que o avaliador identificou cinco aspectos independentes: rugosidade ( $\mathrm{R}$ - do inglês roughness), soprosidade (B - breathiness), astenia ( $\mathrm{A}$-asteny), tensão (S - strain) e instabilidade (I - instability) para avaliação do grau global da disfonia pela identificação da contribuição de cinco fatores independentes, a saber: rugosidade, soprosidade, astenia, tensão e instabilidade ${ }^{8}$.

Para a análise da Qualidade de vida foi utilizado o protocolo "Qualidade de Vida em Voz" (QVV), que analisa o impacto da disfonia na qualidade de vida do sujeito segundo 10 itens, divididos em três domínios: sócio- 
emocional (questões 4, 5, 8 e 10), físico (questões 1, 2, 3, 6,7 e 9) e global (todas as questões) ${ }^{9}$.

Para a análise da desvantagem vocal foi utilizado o protocolo "Índice de Desvantagem Vocal - 10" (IDV- 10). Este protocolo produz um escore total único, calculado por somatória simples das respostas de seus itens, podendo variar de 0 a 40 pontos, sendo 0 o indicativo de nenhuma desvantagem e 40, de desvantagem máxima ${ }^{10}$.

Para a análise da fadiga vocal foi utilizado o protocolo "Índice de Fadiga Vocal" (IFV) composto por 19 questões, divididas em 3 categorias, sendo 15 sobre "Fadiga e restrição vocal", 5 sobre o "Desconforto físico associado à voz" e 3 quanto a "Recuperação com repouso vocal"11.

Para a análise de sintomas de ansiedade e depressão foram utilizados dois questionários de auto-relato também validados - Inventário Beck de Depressão (Beck Depression Inventory - BDI) e o Inventário Beck de Ansiedade (Beck Anxiety Inventory - BAI). O BDI avalia sintomas característicos de depressão, discriminando graus de intensidade dos sintomas. O inventário é composto por 21 categorias de sintomas e atitudes, relacionados à presença de manifestações comportamentais, cognitivas, afetivas e somáticas de sintomas depressivos, incluindo humor, tristeza, pessimismo, sentimentos de fracasso, insatisfação, sentimento de culpa, sentimentos de punição, autodepreciação, auto-acusação, desejo de auto-punição, crises de choro, irritabilidade, isolamento social, indecisão, inibição no trabalho, distúrbios do sono, fatigabilidade, 
perda de apetite, perda de peso, preocupações somáticas e perda da libido ${ }^{12}$. O BAI avalia sintomas característicos de ansiedade e é composto de 21 itens relacionados à presença de sintomas ansiosos ${ }^{13}$.

\section{Análise Estatística}

As análises estatísticas dos dados foram realizadas por meio do programa Statistical Package for Social Science (SPSS), utilizando análise descritiva e de correlação, teste de Wilcoxon para comparação dos dados de auto-avaliação e análise da qualidade vocal, teste McNemar e Kappa para análise qualitativa bivariada. O nível de significância adotado foi de $5 \%(p=0,05)$ e intervalo de confiança de $95 \%$.

\section{RESULTADOS}

A amostra deste estudo foi composta por 15 sujeitos, 9 (60\%) do gênero masculino e 6 (40\%) do gênero feminino, na faixa etária de 18 a 63 anos (idade mediana $37,0 \pm 13,2)$ com idade no diagnóstico de EM entre 16 a 50 anos (mediana de $25,0 \pm 11,1$ ). Com relação ao nível de escolaridade, três $(n=3)$ sujeitos tinham ensino fundamental incompleto, um $(n=1)$ ensino médio incompleto, sete $(n=7)$ ensino médio completo e quatro $(n=4)$ ensino superior completo. A maioria dos sujeitos apresentou incapacidade funcional leve (EDSS $\leq 4)$. Dos sujeitos estudados, 14 apresentavam curso da doença do 
tipo recorrente-remitente $(93 \%)$ e 1 secundariamenteprogressiva (7\%). Um dos indivíduos foi excluído do estudo por não preencher os critérios de inclusão estabelecidos, permanecendo 14 sujeitos para serem reavaliados a partir do T2 até o final do estudo. Os dados demográficos dos sujeitos estão apresentados na Tabela 1.

Tabela 1. Dados demográficos e clínicos dos 15 sujeitos com esclerose múltipla.

\begin{tabular}{lccc}
\hline & Idade & $\begin{array}{c}\text { Numero de } \\
\text { Surtos }\end{array}$ & $\begin{array}{c}\text { Idade no } \\
\text { Diagnostico }\end{array}$ \\
\hline Média & 37,4 & 2,5 & 30,5 \\
\hline Mediana & 37,0 & 3,0 & 25,0 \\
\hline Desvio Padrão & $\pm 13,1$ & $\pm 1,3$ & $\pm 11,0$ \\
\hline Faixa (Min - Max) & $18-63$ & $1-5$ & $16-50$ \\
\hline
\end{tabular}

A Tabela 2 apresenta os valores das variáveis qualitativas obtidas nos dois tempos da análise.

$\mathrm{Na}$ análise bivariada foi observada diferença, com permanência do quadro de limitação $(p=0,016)$ e fala lenta $(p=0,031)$ após os cinco meses. Na reavaliação (T2), os sujeitos passaram a apresentar sintomas sensitivos, o que não foi observado no primeiro contato (T1; Tabela 3; $p=0,134)$.

Para avaliar a confiabilidade dos dados, foram utilizados o teste de Wilcoxon e o teste de análise de correlação. Observa-se na Tabela 4 que as correlações entre IFV escore recuperação com repouso vocal, G, R e B não foram significantes $(p=0,967)$. 
Tabela 2. Análise qualitativa univariada dos 15 sujeitos no primeiro tempo (T1) e após 5 meses (T2) com 14 sujeitos.

\begin{tabular}{|c|c|c|c|c|c|}
\hline Variável & Categoria & Tempo 1 & $\%$ & Tempo 2 & $\%$ \\
\hline \multirow[t]{2}{*}{ Apresentou surto } & Não & 11 & 73 & 12 & 86 \\
\hline & Sim & 4 & 27 & 2 & 14 \\
\hline \multirow[t]{2}{*}{ Sentiu dor } & Não & 6 & 40 & 6 & 43 \\
\hline & Sim & 9 & 60 & 8 & 57 \\
\hline \multirow{2}{*}{$\begin{array}{l}\text { Sentiu-se } \\
\text { limitado }\end{array}$} & Não & 5 & 33 & 8 & 57 \\
\hline & Sim & 10 & 67 & 6 & 43 \\
\hline \multirow{2}{*}{$\begin{array}{l}\text { Apresentou } \\
\text { dificuldade para } \\
\text { engolir }\end{array}$} & Não & 11 & 73 & 7 & 50 \\
\hline & Sim & 4 & 27 & 7 & 50 \\
\hline \multirow{2}{*}{$\begin{array}{l}\text { Sentiu mudança } \\
\text { na voz }\end{array}$} & Não & 10 & 67 & 6 & 43 \\
\hline & Sim & 5 & 33 & 8 & 57 \\
\hline \multirow{2}{*}{$\begin{array}{l}\text { Acha que a voz } \\
\text { ficou mais rouca } \\
\text { e/ou soprosa }\end{array}$} & Não & 11 & 73 & 10 & 71 \\
\hline & Sim & 4 & 27 & 4 & 29 \\
\hline \multirow{2}{*}{$\begin{array}{l}\text { Sentiu cansaço } \\
\text { ao falar }\end{array}$} & Não & 8 & 53 & 6 & 43 \\
\hline & Sim & 7 & 47 & 8 & 57 \\
\hline \multirow{2}{*}{$\begin{array}{l}\text { Acha que sua voz } \\
\text { ficou mais fraca }\end{array}$} & Não & 7 & 47 & 10 & 71 \\
\hline & Sim & 8 & 53 & 4 & 29 \\
\hline \multirow{2}{*}{$\begin{array}{l}\text { Acha que sua fala } \\
\text { ficou mais lenta }\end{array}$} & Não & 8 & & & \\
\hline & Sim & 7 & $\begin{array}{l}53 \\
47\end{array}$ & $\begin{array}{l}8 \\
6\end{array}$ & $\begin{array}{l}57 \\
43\end{array}$ \\
\hline \multirow{2}{*}{$\begin{array}{l}\text { Senti fadiga ao } \\
\text { falar }\end{array}$} & Não & 6 & 40 & 5 & 36 \\
\hline & Sim & 9 & 60 & 9 & 64 \\
\hline \multirow{2}{*}{$\begin{array}{l}\text { Apresentou } \\
\text { sintomas } \\
\text { sensitivos } \\
\text { (formigamento) }\end{array}$} & Não & 4 & 27 & 10 & 71 \\
\hline & Sim & 11 & 73 & 4 & 29 \\
\hline \multirow[t]{2}{*}{$\begin{array}{l}\text { Usou mais a voz } \\
\text { nesses } \\
\text { Seis meses }\end{array}$} & Não & 15 & 100 & 14 & 100 \\
\hline & Disforia & 1 & 7 & & \\
\hline \multirow{3}{*}{ Depressão } & Grave & 2 & 13 & 2 & 14 \\
\hline & moderada a grave & 1 & 7 & 1 & 7 \\
\hline & sem depressão & 11 & 74 & 11 & 79 \\
\hline \multirow{4}{*}{ Ansiedade } & Grave & 2 & 13 & 2 & 14 \\
\hline & Leve & 3 & 20 & 3 & 21 \\
\hline & Mínimo & 8 & 53 & 4 & 29 \\
\hline & Moderado & 2 & 13 & 5 & 36 \\
\hline
\end{tabular}


Tabela 3. Análise qualitativa bivariada dos sujeitos no primeiro tempo (T1) e após 5 meses (T2). Surto: teve surto; Dor: sentiu dor; Limitação: se sentiu limitado; Disfagia: apresentou dificuldade para engolir; Mudança na voz: apresentou mudança na voz; Rouca soprosa: acha que a voz ficou mais rouca e/ou soprosa; cansaço: senti cansaço para falar; fraca: acha que a voz ficou mais fraca; lenta: acha que a fala ficou mais lenta; fadiga: senti fadiga ao falar; sintomas sensitivos: apresentou sintomas de sensibilidade; depressão: sintomas clínicos; ansiedade: sintomas clínicos.

\begin{tabular}{|c|c|c|c|c|c|c|}
\hline \multirow[t]{2}{*}{ Surto - T1 } & \multicolumn{2}{|c|}{ Surto - T2 } & \multirow[b]{2}{*}{ Total } & \multirow[t]{2}{*}{ McNemar } & \multirow[t]{2}{*}{ Kappa } & \multirow[t]{2}{*}{ p-valor } \\
\hline & não & $\operatorname{sim}$ & & & & \\
\hline Não & 9 & 1 & 10 & \multirow{3}{*}{0,625} & \multirow{3}{*}{0,176} & \multirow{3}{*}{0,469} \\
\hline Sim & 3 & 1 & 4 & & & \\
\hline Total & 12 & 2 & 14 & & & \\
\hline \multirow{2}{*}{ Dor - T1 } & \multicolumn{2}{|c|}{ Dor - T2 } & & \multirow[t]{2}{*}{ McNemar } & \multirow[t]{2}{*}{ Kappa } & \multirow[t]{2}{*}{ p-valor } \\
\hline & não & $\operatorname{sim}$ & Total & & & \\
\hline Não & 3 & 3 & 6 & \multirow[t]{3}{*}{1,000} & 0,125 & \multirow{3}{*}{0,640} \\
\hline \multirow[t]{2}{*}{ Sim } & 3 & 5 & 8 & & & \\
\hline & 6 & 8 & 14 & & & \\
\hline \multirow{2}{*}{ Limitação - T1 } & \multicolumn{2}{|c|}{ Limitação T2 } & & \multirow[t]{2}{*}{ McNemar } & \multirow[t]{2}{*}{ Kappa } & \multirow[t]{2}{*}{ p-valor } \\
\hline & não & Sim & Total & & & \\
\hline Não & 5 & 0 & 5 & \multirow{3}{*}{0,250} & \multirow{3}{*}{0,588} & \multirow{3}{*}{0,016} \\
\hline \multirow[t]{2}{*}{ Sim } & 3 & 6 & 9 & & & \\
\hline & 8 & 6 & 14 & & & \\
\hline Disfagia - T1 & Dist & $a-T 2$ & & McNemar & Kappa & p-valor \\
\hline & não & Sim & Total & & & \\
\hline Não & 5 & 5 & 10 & 0,453 & 0,000 & 1,000 \\
\hline Sim & 2 & 2 & 4 & & & \\
\hline & 7 & 7 & 14 & & & \\
\hline Mudanca - T1 & Mud & $a-T 2$ & & McNemar & Kappa & p-valor \\
\hline & não & Sim & Total & & & \\
\hline Não & 5 & 4 & 9 & 0,357 & 0,314 & 0,198 \\
\hline Sim & 1 & 4 & 5 & & & \\
\hline & 6 & 8 & 14 & & & \\
\hline Rouca - T1 & Ro & $-\mathbf{T 2}$ & & McNemar & Kappa & p-valor \\
\hline & não & Sim & Total & & & \\
\hline Não & 8 & 2 & 10 & 1,000 & 0,300 & 0,262 \\
\hline Sim & 2 & 2 & 4 & & & \\
\hline & 10 & 4 & 14 & & & \\
\hline Cansaço - T1 & Can & $0-T 2$ & & McNemar & Kappa & p-valor \\
\hline & não & Sim & Total & & & \\
\hline Não & 4 & 3 & 7 & 1,000 & 0,286 & 0,280 \\
\hline Sim & 2 & 5 & 7 & & & \\
\hline & 6 & 8 & 14 & & & \\
\hline Fraca - T1 & & $-\mathrm{T2}$ & & McNemar & Kappa & p-valor \\
\hline & não & Sim & Total & & & \\
\hline Não & 5 & 1 & 6 & 0,219 & 0,192 & 0,393 \\
\hline Sim & 5 & 3 & 8 & & & \\
\hline
\end{tabular}




\begin{tabular}{|c|c|c|c|c|c|c|c|c|}
\hline \multirow{2}{*}{ Lenta - T1 } & \multicolumn{2}{|c|}{ Lenta - T2 } & \multirow[b]{2}{*}{ Total } & \multirow[t]{2}{*}{ McNemar } & \multirow[t]{2}{*}{ Kappa } & \multirow[t]{2}{*}{ p-valor } & & \\
\hline & não & Sim & & & & & & \\
\hline Não & 6 & 1 & 7 & 1,000 & 0,571 & 0,031 & & \\
\hline \multirow[t]{2}{*}{ Sim } & 2 & 5 & 7 & & & & & \\
\hline & 8 & 6 & 14 & & & & & \\
\hline \multirow[t]{2}{*}{ Fadiga - T1 } & \multicolumn{2}{|c|}{ Fadiga - T2 } & & McNemar & Kappa & p-valor & & \\
\hline & não & sim & Total & & & & & \\
\hline Não & 2 & 3 & 5 & 1,000 & 0,067 & 0,803 & & \\
\hline \multirow[t]{2}{*}{ Sim } & 3 & 6 & 9 & & & & & \\
\hline & 5 & 9 & 14 & & & & & \\
\hline \multirow{2}{*}{$\begin{array}{c}\text { Sensitivos - } \\
\text { T1 }\end{array}$} & \multicolumn{2}{|c|}{ Sensitivos - T2 } & & McNemar & Kappa & p-valor & & \\
\hline & não & sim & Total & & & & & \\
\hline Não & 4 & 0 & 4 & 0,031 & 0,276 & 0,134 & & \\
\hline \multirow[t]{2}{*}{ Sim } & 6 & 4 & 10 & & & & & \\
\hline & 10 & 4 & 14 & & & & & \\
\hline \multirow{2}{*}{$\begin{array}{c}\text { Depressão - } \\
\text { T1 }\end{array}$} & \multicolumn{3}{|c|}{ Depressão - T2 } & & $\begin{array}{c}\text { McNema } \\
\mathbf{r}\end{array}$ & Kappa & $\begin{array}{c}\text { p- } \\
\text { valor }\end{array}$ & \\
\hline & $\begin{array}{c}\text { grav } \\
\text { e }\end{array}$ & $\begin{array}{l}\text { moderad } \\
\text { o a grave }\end{array}$ & $\begin{array}{c}\text { Sem } \\
\text { depressã } \\
0 \\
\end{array}$ & Total & & & & \\
\hline Disforia & 0 & 1 & 0 & 1 & & & & \\
\hline Grave & 2 & 0 & 0 & 2 & & & & \\
\hline $\begin{array}{l}\text { moderada a } \\
\text { grave }\end{array}$ & 0 & 0 & 1 & 1 & & & & \\
\hline \multirow[t]{2}{*}{ sem depressão } & 0 & 0 & 10 & 10 & & & & \\
\hline & 2 & 1 & 11 & 14 & & & & \\
\hline \multirow{2}{*}{$\begin{array}{c}\text { Ansiedade - } \\
\text { T1 }\end{array}$} & \multicolumn{4}{|c|}{ Ansiedade - T2 } & & \multirow[t]{2}{*}{$\begin{array}{c}\text { McNema } \\
\mathbf{r}\end{array}$} & \multirow[t]{2}{*}{ Kappa } & \multirow[t]{2}{*}{$\begin{array}{c}\text { p- } \\
\text { valor }\end{array}$} \\
\hline & $\begin{array}{c}\text { grav } \\
\text { e }\end{array}$ & leve & mínimo & $\begin{array}{c}\text { moderad } \\
0\end{array}$ & Total & & & \\
\hline Grave & 1 & 0 & 0 & 1 & 2 & \multirow{5}{*}{0,406} & 0,131 & 0,371 \\
\hline Leve & 0 & 0 & 1 & 2 & 3 & & & \\
\hline Mínimo & 0 & 3 & 3 & 1 & 7 & & & \\
\hline \multirow[t]{2}{*}{ moderado } & 1 & 0 & 0 & 1 & 2 & & & \\
\hline & 2 & 3 & 4 & 5 & 14 & & & \\
\hline
\end{tabular}

Houve correlação entre os protocolos IDV $(r=0,675$; $p=0,008)$, QVV escore total $(r=0,829 ; p=0,000)$, QVV escore sócio-emocional ( $r=0,650 ; p=0,012)$, QVV escore do funcionamento físico $(r=0,832 ; p=0,000)$, IFV escore Fadiga e restrição vocal $(r=0,886 ; p=0,000)$, IFV escore desconforto físico associado à voz $(r=0,733 ; p=0,003)$ e na avaliação perceptiva auditiva nos domínios Astenia 
Instabilidade $(r=849 ; p=0,000)$.

Tabela 4. Análise de correlação das variáveis no primeiro tempo (T1) e após 5 meses (T2), utilizando o teste de Wilcoxon.

\begin{tabular}{|c|c|c|c|c|c|c|c|c|c|c|}
\hline \multirow[b]{2}{*}{ Variável } & \multirow[b]{2}{*}{ Média } & \multirow[b]{2}{*}{$\mathrm{N}$} & \multirow[b]{2}{*}{ DP } & \multirow[b]{2}{*}{ correlação } & \multirow[b]{2}{*}{$\begin{array}{c}\mathrm{p}- \\
\text { valor }\end{array}$} & \multicolumn{2}{|c|}{$\begin{array}{c}\text { Diferenças } \\
\text { pareadas }\end{array}$} & \multirow[b]{2}{*}{$\mathrm{t}$} & \multirow[b]{2}{*}{$\begin{array}{c}\mathrm{p}- \\
\text { valor }\end{array}$} & \multirow{2}{*}{$\begin{array}{l}\text { Wilcoxon } \\
\text { p-valor }\end{array}$} \\
\hline & & & & & & média & DP & & & \\
\hline $\begin{array}{l}\text { IDV - T1 } \\
\text { IDV - T2 }\end{array}$ & $\begin{array}{c}9,6 \\
13,6\end{array}$ & $\begin{array}{l}14 \\
14\end{array}$ & $\begin{array}{c}8,4 \\
12,3\end{array}$ & 0,675 & 0,008 & $-4,0$ & 9,1 & $-1,65$ & 0,123 & 0,050 \\
\hline $\begin{array}{l}\text { QVVet - } \\
\text { T1 }\end{array}$ & 76,6 & 14 & 28,5 & 0,829 & 0,000 & 7,7 & 16,7 & 1,72 & 0,109 & 0,120 \\
\hline $\begin{array}{l}\text { QVVet - } \\
\text { T2 }\end{array}$ & 68,9 & 14 & 28,6 & & & & & & & \\
\hline $\begin{array}{l}\text { QVVse - } \\
\text { T1 }\end{array}$ & 84,8 & 14 & 28,6 & 0,650 & 0,012 & 7,6 & 24,2 & 1,18 & 0,260 & 0,262 \\
\hline $\begin{array}{l}\text { QVVse - } \\
\text { T2 }\end{array}$ & 77,2 & 14 & 29,2 & & & & & & & \\
\hline $\begin{array}{l}\text { QVVf - T1 } \\
\text { QVVf - T2 }\end{array}$ & $\begin{array}{l}71,1 \\
63,4\end{array}$ & $\begin{array}{l}14 \\
14\end{array}$ & $\begin{array}{l}30,1 \\
29,8\end{array}$ & 0,832 & 0,000 & 7,7 & 17,4 & 1,66 & 0,120 & 0,093 \\
\hline $\begin{array}{l}\text { IFVfrv - } \\
\text { T1 }\end{array}$ & 14,9 & 14 & 12,8 & 0,886 & 0,000 & $-5,0$ & 6,0 & $-3,12$ & 0,008 & 0,010 \\
\hline $\begin{array}{l}\text { IFVfrv - } \\
\text { T2 }\end{array}$ & 19,9 & 14 & 12,0 & & & & & & & \\
\hline $\begin{array}{l}\text { IFVdfav - } \\
\text { T1 }\end{array}$ & 3,5 & 14 & 4,5 & 0,733 & 0,003 & $-1,8$ & 3,4 & $-1,96$ & 0,071 & 0,051 \\
\hline $\begin{array}{l}\text { IFVdfav - } \\
\text { T2 }\end{array}$ & 5,3 & 14 & 4,8 & & & & & & & \\
\hline $\begin{array}{l}\text { IFVrrv - } \\
\text { T1 }\end{array}$ & 9,7 & 14 & 3,3 & 0,012 & 0,967 & $-1,2$ & 4,6 & $-0,98$ & 0,344 & 0,202 \\
\hline $\begin{array}{l}\text { IFVrrv - } \\
\text { T2 }\end{array}$ & 10,9 & 14 & 3,2 & & & & & & & \\
\hline $\mathrm{G}-\mathrm{T} 1$ & 1,3 & 14 & 0,5 & & & & & & & 1,000 \\
\hline $\mathrm{G}-\mathrm{T} 2$ & 1,3 & 14 & 0,5 & & & & & & & \\
\hline $\mathrm{R}-\mathrm{T} 1$ & 0,9 & 14 & 0,7 & & & & & & & 1,000 \\
\hline $\mathrm{R}-\mathrm{T} 2$ & 0,9 & 14 & 0,7 & & & & & & & \\
\hline $\mathrm{B}-\mathrm{T} 1$ & 0,9 & 14 & 0,7 & & & & & & & 1,000 \\
\hline$B-T 2$ & 0,9 & 14 & 0,7 & & & & & & & \\
\hline$A-T 1$ & 0,4 & 14 & 0,8 & 0,742 & 0,002 & $-0,2$ & 0,6 & $-1,38$ & 0,189 & 0,180 \\
\hline$A-T 2$ & 0,6 & 14 & 0,8 & & & & & & & \\
\hline $\begin{array}{l}S-T 1 \\
S-T 2\end{array}$ & $\begin{array}{l}0,3 \\
0,2\end{array}$ & $\begin{array}{l}14 \\
14\end{array}$ & $\begin{array}{l}0,5 \\
0,4\end{array}$ & 0,826 & 0,000 & 0,1 & 0,3 & 1,00 & 0,336 & 0,317 \\
\hline $\mathrm{I}-\mathrm{T} 1$ & 0,7 & 14 & 0,5 & & & 0,1 & 0,3 & 1,00 & 0,336 & 0,317 \\
\hline $\mathrm{I}-\mathrm{T} 2$ & 0,6 & 14 & 0,5 & 0,849 & 0,000 & & & & & \\
\hline
\end{tabular}

IDV: Índice de Desvantagem Vocal; QVVet: Qualidade de vida em Voz escore total; QVVse: Qualidade de vida em Voz sócio-emocional; QVVf: Qualidade de Vida em Voz funcionamento físico; IFVfrv: Índice de Fadiga Vocal fadiga e restrição vocal;IFVdfav: Índice de Fadiga Vocal desconforto físico associado à voz; IFVrrv:Índice de Fadiga Vocal recuperação com repouso vocal; G: Grau geral; R: Rugosidade; B: Soprosidade; A: Astenia; S: Tensão; I: Instabilidade. 


\section{DISCUSSÃO}

A análise demográfica mostra que indivíduos com EM apresentam características semelhantes às descritas em estudos anteriores. Dos sujeitos estudados, 14 (60\%) apresentam forma recorrente-remitente e apenas um na forma secundariamente progressiva. Em nossa amostra 0 predomínio maior foi do gênero masculino (60\%), comparado a outros estudos com prevalência de $61 \%$ dos sujeitos do gênero feminino ${ }^{14}$.

Os resultados do nosso estudo revelam ainda que a pontuação obtida nos protocolos IDV, QVV escore total, QVV escore sócio-emocional e QVV escore do funcionamento físico apresentam correlação positiva, sugerindo que a qualidade de vida relacionada à voz e o índice de desvantagem pioram nesses sujeitos com 0 tempo. Em um estudo que avaliaram 87 sujeitos (59 com EM e 27 como grupo controle), 52,5\% apresentaram fadiga com base no escore de corte, comparados com 3,57\% do grupo controle, sete dos 59 indivíduos apresentaram sintomas vocais em comparação com três sujeitos do grupo controle $^{14}$. Outro estudo realizado com 36 sujeitos com EM e 36 do grupo controle, tinha como objetivo avaliar as alterações vocais subjetivas utilizando o Protocolo Índice de Desvantagem Vocal (IDV), Questionário de Qualidade de Vida em Voz (QVV) quanto alterações perceptivas da voz utilizando a escala GRBASI e os resultados obtidos mostraram uma forte correlação entre as escalas IDV e QVV $(r=0,87)$ e, que a média do IDV foi maior no grupo de 
pacientes. O aspecto físico do QVV foi avaliado com maior pontuação, seguido pelos aspectos funcionais e emocionais. De acordo com a escala GRBASI, as diferenças entre os dois grupos foram significantes para os itens grau geral, rugosidade, soprosidade e tensão ${ }^{15}$.

Os resultados de nossa pesquisa apontaram que os sujeitos com EM apresentaram fadiga vocal e limitação em suas atividades de vida diária no protocolo de autoavaliação. Em um estudo com 61 sujeitos, que teve como objetivo determinar o efeito das limitações funcionais e da fadiga na qualidade de vida em pessoas com EM, obteve como resultado valores significantes para a associação do funcionamento físico com as variáveis EDSS, fadiga, tônus de membro inferior e marcha e, que essas incapacidades são fatores independentes que afetam negativamente a qualidade de vida em pessoas com $\mathrm{EM}^{16}$. Em outro estudo com 40 sujeitos (36 do tipo recorrente-remitente, 3 progressivas primárias e três progressivas secundárias) comparados a um grupo controle com 42 indivíduos tinha como objetivo analisar a prevalência de sintomas vocais em sujeitos com EM, medir seus parâmetros acústicos e descrever a correlação entre os achados, a duração e estagio da doença, bem como o grau de incapacidade. Os sintomas vocais mais frequentes foram quebras de voz e fadiga vocal presente em 10 de 40 sujeitos, enquanto a rouquidão estava presente em apenas dois indivíduos ${ }^{17}$. Ainda, em outro estudo com 87 sujeitos, sendo 59 com EM e 27 como grupo controle investigou a qualidade de vida 
relacionada à voz em um grupo de sujeitos com EM. Os resultados mostraram que $52,5 \%$ dos sujeitos e 3,57\% dos controles apresentaram fadiga com corte de 4 na escala de gravidade da fadiga (FSS) ${ }^{14}$.

$\mathrm{Na}$ análise perceptivo-auditiva, nosso estudo apresentou mudanças vocais nos parâmetros de astenia, tensão e instabilidade (Tabela 4). Como observado em um estudo de auto avaliação da voz comparado com os resultados da avaliação perceptiva auditiva em sujeitos com EM, em que foram comparados 38 sujeitos com EM a 38 sujeitos saudáveis, mostrando que os sujeitos com EM apresentam IDV significantemente maior que os participantes do grupo controle. Na escala GRBASI, os pesquisadores estabeleceram um nível de disfonia (grau geral $=55 \%$, rugosidade e soprosidade $=66 \%$, astenia $=34 \%$ e tensão=55\%). $34 \%$ dos participantes tiveram um componente da Escala GRBASI classificada como grave ${ }^{18}$. Em outro estudo, com 27 sujeitos com EM e 27 para grupo controle, demonstraram que a escala GRBASI revelou maiores valores de astenia e tensão para sujeitos com esclerose múltipla em comparação com grupo controle ${ }^{19}$.

Observou-se que, a despeito da mudança vocal ocorrer no T2 detectado por meio de avaliação formal perceptivo-auditiva, o sujeito não apresenta auto relato de que observa modificação na sua voz após 5 meses de evolução da doença. Este fato reforça a necessidade de que o sujeito tenha acesso ao atendimento de profissionais com expertise na assistência em voz. Além disso, por se tratar 
de uma doença com características de progressivas com evolução crônica, o paciente pode não perceber ou se habituar com o novo padrão vocal que se manifesta no curso natural da doença e por isso, não relatar modificações na voz.

$\mathrm{Na}$ análise qualitativa bivariada, constatou que não houve associação entre as escalas de sintomas de alteração de humor e modificação na qualidade vocal utilizando como referência os protocolos IDV e QVV. Em um estudo que comparou três grupos de sujeitos (Síndrome Clínica Isolada, Grupo Controle e EM) verificou que houve diferença significativa nos quatros pontos de corte do BAI entre os pacientes controles e EM. Na aplicação do BDI quando comparado os três grupos (controle, CIS, EM), mostraram diferença significativa entre o grupo controle versus EM. Os resultados corroboram a visão de que sujeitos com esclerose múltipla têm mais sintomas de depressão e ansiedade do que o grupo controle ${ }^{20}$.

Ainda com relação aos sintomas de depressão e ansiedade, outro estudo realizado com 189 sujeitos, com finalidade de relacionar a relação entre ansiedade e depressão e relevância do processamento emocional, equilíbrio emocional e enfrentamento para sintomatologia depressiva obteve como resultado que 16 participantes tiveram ansiedade e depressão, 8 apresentaram sintomas de ansiedade, 37 apresentaram sintomas de depressão e 128 não apresentaram estes sintomas. Neste mesmo estudo os resultados mostraram uma perturbação do 
equilíbrio emocional e do bem-estar duas vezes maior que os das demais escalas, sugerindo que essas eram as estratégias de enfrentamento mais comum, usado pelos entrevistados ${ }^{21}$.

Algumas limitações devem ser destacadas em nosso estudo como o tamanho da amostra reduzido, a diversidade de idade e tempo de diagnóstico para entrada e inclusão no estudo. Ainda, o protocolo proposto de avaliação de ansiedade e depressão são inventários de rastreio que por ventura podem não ser sensíveis a uma alteração de humor melhor diagnosticada se houvesse avaliação clínica psiquiátrica completa. Com isso, se faz necessário mais estudos na área para investigação da qualidade vocal destes sujeitos e correlação mais robusta dos dados, a exemplo de estudos com universo amostral maior, protocolos de causa-efeito e tratamento estatístico específico de sensibilidade, especificidade e risco, seriam importantes para afirmações assertivas de que as modificações na qualidade vocal são fatores que aumentam o risco para transtorno de humor.

\section{CONCLUSÃO}

A partir da análise dos sujeitos da pesquisa, observase que a despeito da falta de percepção do sujeito na sua piora vocal, foi detectado que há alteração vocal principalmente manifestada por astenia, tensão e instabilidade. 
Os sujeitos com EM apresentam impacto na qualidade de vida em voz, na fadiga vocal nos domínios fadiga e restrição vocal e desconforto físico associado à voz, e piora da desvantagem vocal ao longo do curso da doença.

Não houve presença nem piora de sintomas de depressão e ansiedade nos sujeitos do nosso estudo.

\section{AGRADECIMENTOS}

A toda equipe do Ambulatório de Neurologia do HUCAM, equipe do Núcleo de Voz do espírito Santo (VozES) e em especial os grandes amigos (pacientes).

\section{REFERÊNCIAS}

1.O'Connor P, Canadian Multiple Sclerosis Working Group. Key issues in the diagnosis and treatment of multiple sclerosis. An overview. Neurology 2002;59(6 Supl 3):1-33.

http://dx.doi.org/10.1212/wnl.59.6_suppl_3.s1

2.Moreira MA, Tilbery CP, Lana-Peixoto MA, Mendes MF, Kaimen Maciel DR, Callegaro D. Aspectos históricos de La esclerosis múltiple. Rev Neurol 2002;34:378-83.

https://doi.org/10.33588/rn.3404.2001313

3. Kurtzke JF. A reassessment of the distribution of multiple sclerosis. Acta Neurol Scan 1975;51:110-57.

4.Callegaro D, Goldbaum M, Morais L. The prevalence of multiple sclerosis in the city of São Paulo, Brazil. Acta Neurol Scand 2001; 104:208-13.

https://doi.org/10.1111/j.1600-0404.1975.tb01365.x

5.Lana-Peixoto MA, Frota E, Campos GB, Monteiro LP on behalf of the Brazilian Committee for Treatment and Research in Multiple Sclerosis. The prevalence of multiple sclerosis in Belo Horizonte, Brazil. Arq Neuropsiquiatr 2012;70:102-7.

http://dx.doi.org/10.1590/S0004-282X2012000200006

6.Rocha F, Herreira L, Morales RR. Multiple Sclerosis in Botucatu, Brazil - a population study. Mult Scler 2002;8(supl1):41.

7.Miller JR. Esclerose Múltipla. In: Rowland LP. Merritt Tratado de Neurologia. 10 ed. Rio de Janeiro: Guanabara Koogan; 2002, 887p. 
8.Behlau M. Voz - O Livro do Especialista. vol 1. Rio de Janeiro: Revinter, 2001, 348p.

9.Fabrício MZ, Kasama ST, Martinez EZ. Qualidade de vida relacionada à voz de professores universitários. Rev CEFAC 2010;12:280-7.

http://dx.doi.org/10.1590/S1516-18462009005000062.

10.Costa T, Oliveira G, Behlau M. Validação do Índice de Desvantagem Vocal: 10 (IDV-10) para o português brasileiro. CoDAS 2013;25:482-5.

http://dx.doi.org/10.1590/S2317-17822013000500013

11.Zambon F, Moreti F, Nanquim C, Behlau M. Equivalência cultural da versão brasileira do Índice de Fadiga Vocal - VFI. CoDAS 2017; 29:e20150261. http://dx.doi.org/10.1590/2317-1782/20172015261

12. Beck AT, Ward CH, Mendelson M, Mock J, Erbaugh G. An inventory for measuring depression. Arch Gen Psych 1961;4:561-71.

http://dx.doi.org/10.1001/archpsyc.1961.01710120031004

13. Beck AT, Brown G, Epstein N, Steer RA. An inventory for measuring clinical anxiety: psychometric properties. J Consult Clin Psychol 1988;56:893-7. http://dx.doi.org/10.1037//0022006x.56.6.893

14. Hamdan AL, Farhat S, Saadeh R, El-Dahouk L, Sibai A, Yamout B. Voice-Related Quality Lify in Patients With Multiple Sclerosis. Autoimmune Dis 2012;2012:143813.

http://dx.doi.org/10.1155/2012/143813

15.Bauer V, Alleric Z, Jancic E, Knezevc B, Prpic D, Kacavenda A. Subjective and perceptual analysis of voice quality and relationship with neurological disfunction in multiple sclerosis patients. Clin Neurol Neurosurg 2013;115 (Suppl 1):S17-20.

http://dx.doi.org/10.1016/j.clineuro.2013.09.015

16. Nogueira LAC, Nóbrega FR, Lopes KN, Thuler LCS, Alvarenga MP. The effect of functional limitations and fatigue on the quality of life in people with multiple sclerosis. Arq Neuropsiquiatr 2009;67:812-7. http://dx.doi.org/10.1590/s0004-282×2009000500006

17.Yamout B, Fuleihan N, Hajj T, Sibai A, Sabra O, Rifai H, et al. Vocal symptoms and acoustic changes in relation to the expanded disability status scale, duration and stage of disease in patients with multiple sclerosis. Eur Arch Otorhinolaryngol 2009;266:1759-65. http://dx.doi.org/10.1007/s00405-009-1003-y

18.Bauer V, Aleric Z, Jancic E. Comparing Voice Self-Assessment with Auditory Perceptual Analysis in Patients with Multiple Sclerosis. Int Ach Otorhinolaryngol 2015;19:100-5. http://dx.doi.org/10.1055/s0034-1397332

19.Dogan M, Midi I, Yazici MA, Kocak I, Gunal D, Sehitoglu MA. Objective and subjective evaluation of voice quality in multiple sclerosis. J Voice 2007;21:735-40.

http://dx.doi.org/10.1016/j.jvoice.2006.05.006

20.Anhoque CF, Domingues SCA, Carvalho T, Teixeira AL, Domingues RB. Anxiety and depressive symptoms in clinically isolated syndrome 
and multiple sclerosis. Arq Neuropsiquiatr 2011;69:882-6. http://dx.doi.org/10.1590/s0004-282x2011000700006

21.Gay MC, Bungener C, Thomas S, Vrignaud P, Thomas PW, Baker $\mathrm{R}$, et al. Anxiety, emotional processinng and depression in people with multiple sclerosis. BMC Neurology 2017;17:43. http://dx.doi.org/10.1186/s12883-017-0803-8 